\title{
A Systematic Approach to Develop a Family of Theories for Structural Elements
}

\author{
Peter D. Folkow \\ Chalmers University of Technology \\ Sweden
}

Modelling of structural elements are of fundamental importance within mechanical engineering. As solutions directly based on 3D theories (e.g. using finite element) are very time consuming in many situations, there are still needs to use approximate theories for beams, plates and shells. The classical theories for such elements are widely used and their range of accuracy is in many situations well known. However, if one wants to model structures beyond these levels e.g. at higher frequencies, there are needs to develop better theories. And, perhaps more important, the development of new advanced materials puts these classical theories to the test. For example when adopting materials that involve several phases (poroelastic, piezoelectric, functionally graded, sandwich) these classical theories do not generally capture the structural behavior in a satisfactory manner.

A remedy is to develop more advanced approximate theories for structural elements. A standard procedure is to apply various sorts of series expansion techniques together with additional simplifications. In the present formulations, rigorous series expansion approaches based on the governing 3D equations are used. This results in hierarchies of equations for structural elements that may be truncated to any order without using additional approximations. Moreover, these sets of equations are being variationally consistent as well as asymptotically correct. Hence, by using the sets of developed lower order structural element theories, new and more accurate equations based on the 3D equations are derived for various sets of material configurations. In addition, by studying high enough orders new benchmark solutions to 3D problems are obtained. 